\title{
Carmegliptin Dihydrochloride
}

National Cancer Institute

\section{Source}

National Cancer Institute. Carmegliptin Dihydrochloride. NCI Thesaurus. Code C81701.

The dihydrochloride salt form of carmeg liptin, a potent, long-acting, selective, orally bioavailable, pyrrolidinone-based inhibitor of dipeptidyl peptidase 4 (DPP-4), with hypoglycemic activity. Carmegliptin shows extensive tissue distribution and is excreted unchanged in the urine and bile. 